\title{
CONVERGENCIA
}

Revistade Ciencias Sociales

\section{¿Modernización de la Educación Superior?}

\author{
Ramón Larrauri Torroella \\ Secretaría de Educación Pública y Bienestar Social del \\ Gobierno del Estado de México
}

\section{Introducción}

La modernización de la educación es un proceso histórico cuya situación durante el régimen anterior no fue más que una etapa, o fue una respuesta a una política gubernamental basada en un programa sexenal de dudosa continuidad. Esta disyuntiva fue elaborada como premisa para orientar la búsqueda de elementos que permitieran construir las descripciones y reflexiones que conforman el presente ensayo.

Antes de entrar en materia, permítaseme que presente algunas ideas preliminares, no para establecer supuestos o plantear hipótesis sino para precisar, al menos para mi, los términos involucrados en lo que será el cuerpo del ensayo: la educación superior y la modernización.

Empecemos por la educación superior ¿Qué es?, ¿Un nivel educativo?, ¿Fenómeno social? ¿Se puede considerar que existe como sistema? Sobre este último punto es un hecho reconocido que en nuestro país las instituciones de educación superior comprenden una gama extraordinariamente heterogénea de planteles entre los que existen universidades federales que incluyen enseñanzas tecnológicas, centros tecnológicos que incluyen enseñanzas humanísticas, escuelas superiores orientadas hacia los estudios contables y administrativos, planteles cuya matrícula no rebasa los 20 alumnos y otras que exceden los 300,000, instituciones que funcionan en edificios arrendados o que tienen campus propios en casi todos los Estados de la República, las que ofrecen una sola carrera hasta las que tienen más de sesenta, las que integran secundarias y bachilleratos y las que sólo ofrecen pos grado, las que realizan investigaciones de frontera en ciertos campos y las que no realizan ninguna, las sostenidas o subsidiadas por la federación o los estados y las financiadas por grupos empresariales o religiosos.

Por ello hablar de un sistema nacional de educación superior resulta un eufemismo bien intencionado pero impreciso. La educación superior tampoco es sólo los niveles que la conforman o las funciones que tiene asignadas. 
En ellos están presentes dimensiones pedagógicas y sociales que interesan no sólo a sus integrantes sino a toda la nación. Qué, cómo, y para qué se enseña son cuestiones presentes en el campo pedagógico; en lo social se podría cuestionar si su fin primordial es semejante al de las empresas "producir", en su caso: profesionales. La modernización, por su parte, fue, además de una propuesta política una necesidad inaplazable, al menos en la visión de Carlos Salinas, que, según él, estaba enmarcada por factores endógenos y exógenos qué abarcaban todos los campos de la vida nacional: el Estado, la economía, la agricultura, la educación, las relaciones iglesias-estado, los procesos electorales, etc.

Modernizar al país estructuralmente es rehacer el pacto social creado como resultado de la revolución de 1910. La modernización salinista no era sólo la "puesta al día", "hacer lo que hacen los más avanzados". Era modificarlo todo, no para que todo siguiera igual, si no para que "dejáramos de ser lo que éramos”. El mundo capitalista desarrollado, neo- liberal, era la imagen que nos servía de modelo y meta, aún cuando se le disfrazara de "liberalismo social".

La modernización de la educación superior para nosotros, tiene dos vertientes. Por un lado está el proceso de planeación y desarrollo de las instituciones de educación superior (IES) que ha sido impulsado por el propio Estado desde la creación de la ANUIES, pero que no siempre ha seguido las pautas pretendidas por el gobierno; por el otro, la política modernizadora del salinato, que explícita o implícitamente tuvo una visión de país y por ende de universidad que intentó imponer por la vía de la concertación algunas veces, pero las más fue por la de la imposición.

En el presente ensayo esas dos vertientes son abordadas en tres apartados concretos. En el primero se describen los documentos que desde nuestro punto de vista caracterizan a la modernización de la educación superior y que hemos denominado Documentos Básicos, ellos son: El Programa Integral para el Desarrollo de la Educación Superior (PROI- DES) el Programa para la Modernización Educativa (PME) y la Estrategia para la Consolidación y Desarrollo del Sistema Nacional de Educación Superior.

En el segundo apartado denominado Significación y Sentido de las propuestas, se hace un análisis interpretativo del sentido que orientó las propuestas y el significado que tienen desde la perspectiva de áreas conceptuales presentes en los documentos empleados. En el tercer apartado y a manera de conclusiones se reflexiona sobre los logros de la modernización de la educación superior empleando para el análisis el desarrollo de tres conceptos denominados "estelares" por sus implicaciones en las propuestas 
y la forma en que se concretaron en las acciones de modernización emprendidas.

\section{Los Documentos Básicos}

\section{A) EL PROIDES}

El Programa Integral para el desarrollo de la educación superior (PROIDES) fue el resultado del trabajo realizado por el SINAPPES (Sistema Nacional Para la Planeación de la Educación Superior), la CONPES (Consejo Nacional para la Planeación de la Educación Superior), las CORPES (Coordinaciones Regionales para la Planeación de la Educación Superior), y las COEPES (Comisiones Estatales para la Planeación de la Educación Superior), que fue dado a conocer por la ANUIES en octubre de 1986. Desde el primer momento fue presentado como el producto de un esfuerzo concertado y consensual de las diferentes instancias gubernamentales y las IES tanto en lo individual como a través de la representación de la ANUIES.

Al PROIDES lo constituyen dos documentos fundamentales "La Estrategia Nacional" y los "Proyectos Nacionales" en ellos quedan expresados "Los elementos de planeación y los quehaceres de alcance nacional."

En el primer documento se presenta un conjunto de elementos estratégicos para atender las necesidades, dificultades y carencias de las IES Los elementos indicados son abordados en forma de objetivos políticas y metas, y de ellos se dice que "tienen carácter indicativo y obligarán a las instituciones de educación superior sólo en la medida en que sean adoptados con tal carácter por cada institución o subsistema educativo"(ANUIES, (1982: 45) los elementos estratégicos abordados son Crecimiento, Recursos $\mathrm{Hu}$ manos, Recursos Económicos, Planeación y Coordinación, Docencia, Investigación, Difusión de la Cultura y Extensión de los Servicios y Apoyo Administrativo.

En este documento el horizonte de tiempo es de largo, mediano y corto plazo. El largo plazo es de 10 años (1987-1996) y respecto a él se plantean los objetivos generales y específicos; el mediano plazo es de 5 años (1987-1991); y el corto plazo de uno o de dos años (1987-1988) y respecto a uno de ellos o a ambos se plantean las políticas y las metas.

Se proponen como fines del PROIDES la satisfacción de las necesidades sociales y tratando de precisar se dice: "entendiéndose por éstas (las necesidades sociales), las culturales, científicas políticas y económicas de la nación mexicana"; el otro "fin primordial "es el mejoramiento de la calidad 
de la educación superior, la que consiste en: "alcanzar una mayor congruencia entre lo planeado y la manera en que se realiza el quehacer académico; entre las necesidades sociales y el conocimiento científico tecnológico y humanístico que imparten las IES; así mismo radica en una mayor adecuación de las condiciones institucionales y de los recursos humanos y materiales; en una preparación idónea del personal académico y administrativo; en una mejor formación de los estudiantes y en un mayor grado de compromiso e identificación de los miembros de la comunidad académica con los fines de la educación superior y los objetivos institucionales." (ANUIES, 1982: 93).

En lo personal se piensa que la calidad de la educación consiste en un principio y por sobre todo lo demás en "la mejor formación de los estudiantes " el resto de factores deben tender a este punto ¿De qué sirve que haya congruencia entre planeación y el quehacer académico si no se considera la formación de los estudiantes? ¿Para qué la preparación idónea del personal académico y administrativo?, etc.

Ocho objetivos generales 50 específicos 102 políticas y 74 metas conforman el contenido concreto de la estrategia nacional del PROIDES De ellas se pueden considerar como más relevantes la regulación del crecimiento de la matrícula y la inducción de la demanda hacia los estudios terminales de la educación media. La búsqueda de mejores niveles de preparación, compromiso, condiciones de trabajo y salariales para todo el personal de las IES. La realización de acciones para diversificar las fuentes de ingreso aunadas a la necesidad de mejorar las condiciones financieras de las IES La orientación de planes y programas de estudio encaminándolos tanto a la adquisición de conocimiento y solución de problemas y necesidades sociales como a satisfacer las necesidades del sector productivo y de servicios. Establecer una adecuada interrelación entre docencia e investigación. Hacer de la investigación una tarea institucional permanente y de alto nivel interrelacionar eficientemente la docencia, la investigación y la difusión de la cultura y la extensión de los servicios institucionalizar la evaluación y la autoevaluación de las IES.

El otro documento del PROIDES denominado "Proyectos Nacionales", contiene una serie de acciones cuya ejecución corresponde a organismos de cobertura nacional para responder a aspectos específicos de la problemática y necesidades detectadas en el diagnóstico nacional de la educación superior, son 24 proyectos agrupados en temáticas específicas que comprenden: Opciones para el crecimiento de las IES. Sistema Nacional de Orientación Educativa. Formación de Personal Académico. Formación de personal Administrativo. Estructura y Procedimiento del financiamiento y del gasto. Requerimientos económicos y disponibilidad de re- cursos. Fuentes com- 
plementarias de financiamiento. Apoyo al SINAPPES. Sistema Nacional de Información para la Educación Superior. Evaluación del Sistema de Educación Superior. Autoevaluación institucional. Evaluación y Seguimiento del PROIDES. Estudios sobre los procedimientos de admisión y acreditación de la educación superior. Eficiencia Terminal, Rezago y Deserción Estudiantil. Coordinación y mejoramiento del posgrado. Opciones de innovación en la docencia. Evaluación de los sistemas de educación abierta. Coordinación y mejoramiento de la investigación. Naturaleza y fines de la difusión de la cultura y extensión de los servicios. Sistemas de Educación Continua. Fomento a la producción e intercambio de material audiovisual. Fomento de los servicios bibliotecarios. Estructura y organización académico administrativa de las IES. Apoyo a la formulación y mejoramiento de la normatividad en las IES.

En términos generales se puede decir que el PROIDES se propone:

Establecer las líneas generales para la planeación del sistema de educación superior coadyuvando en la de las IES.

Dar énfasis a la planeación con horizontes temporales amplios sin perder de vista los requerimientos inmediatos. Hacer hincapié para que la educación superior contribuya con efectividad a las necesidades del desarrollo nacional Atender la necesidad de reorientar el crecimiento de la educación superior, disminuyendo los contrastes entre regiones geográficas; alentando la oferta de carreras en las áreas de las ciencias naturales y exactas, las ingenierías y las tecnologías así como en las humanidades; desalentando la demanda en las áreas administrativa, contable jurídica y médica; y orientando el flujo escolar hacia otras opciones de formación terminal en el nivel medio superior. Fomentar la innovación en la docencia. Atender la urgencia de crear y desarrollar carreras prioritarias, programas de posgrado e investigaciones. Redoblar esfuerzos para la formación y actualización del personal de las IES. Definir criterios claros y mecanismos eficaces para la asignación de los subsidios a las IES. Consolidar la función de la difusión de la cultura y la extensión de los servicios. Innovar y adecuar las estructuras y los procedimientos administrativos.

\section{B) El Programa para la Modernización Educativa (PME)}

El capítulo 7 del Programa propuesto por el gobierno salinista estuvo dedicado a la Educación Superior y de Posgrado e Investigación Científica, Humanística y Tecnológica; partía de la consideración que en este caso la modernización educativa adquiría "el carácter de una acción convergente, resultado de las iniciativas de las autoridades, de los esfuerzos que realice cada institución y de las líneas de concertación adoptadas por los integrantes del sistema de educación superior." (PEF, 1989: 124). 
Las características propuestas para la modernización de este nivel educativo se plantean "con absoluto respeto a la naturaleza jurídica de cada institución y se fundamenta en los trabajos del SINAPPES, "recogiendo las aportaciones del PROIDES y las declaraciones y aportaciones de ANUIES” concretándose en: el ofrecimiento de brindar mayor apoyo a las instituciones de educación superior tecnológica para que consoliden e incrementen la calidad de sus servicios; brindar asesoría a los gobiernos estatales que se proponga abrir nuevas instituciones del tipo tecnológico' respetar el compromiso de las instituciones universitarias de evaluar sistemáticamente su actividad de mejorar la calidad del servicio que ofrecen, de reforzar los mecanismos de actualización, evaluación y promoción del personal académico y de impulsar la cultura científica y "el espíritu de solidaridad social." Propone como objetivos centrales "Mejorar la calidad de la educación superior para formar los profesionales que requiere el desarrollo nacional."(PEF, 1989: 130) Atender la demanda de educación superior "asegurando la oportunidad de ingreso a los estudiantes que procedan de las regiones y grupos sociales más desfavorecidos, con aptitudes para cursar estudios de nivel superior." Vincular a las instituciones con la sociedad y fortalecer el sistema de coordinación y planeación nacional de educación superior .Dentro de las estrategias planteadas se destacan la decisión del Gobierno Federal de hacer del SINAPPES el instrumento para la consolidación del sistema nacional de educación superior. Definir conjuntamente con las IES criterios claros y mecanismos eficientes para la asignación de recursos. Modernizar la normatividad que la regula. Como metas principales se pueden apuntar, en el área tecnológica: Diseñar y concertar la descentralización de la educación superior tecnológica. Iniciar un sistema nacional de acreditación por suficiencia del conocimiento. Consolidar y ampliar la modalidad abierta. Actualizar instalaciones y equipo de talleres y laboratorios. En el área universitaria instalar la comisión nacional de evaluación de la E.S. Iniciar el proceso de organización de la universidad nacional abierta. Promover la organización de un sistema nacional de Bibliotecas de E.S.

\section{C). Consolidación y desarrollo del Sistema Nacional de Educación Superior.}

Como resultado de su XXIII Reunión Ordinaria, la Asamblea General de la ANUIES publicó en febrero de 1990 el documento denominado "Consolidación y desarrollo del sistema nacional de educación superior.," De él, resulta de especial interés para este ensayo el capitulo que aborda la posición de la ANUIES ante el programa de la modernización educativa y los cinco capítulos de la segunda parte en donde se presenta la estrategia de la 
ANUIES para mejorar y consolidar el Sistema Nacional de Educación Superior .La ANUIES plantea una serie de reflexiones sobre, las propuestas generales y específicas del Programa para la Modernización Educativa que se pueden sintetizar en los siguientes puntos:

La educación superior no debe ser descuidada por el hecho que el Estado otorgue atención prioritaria a la educación primaria, atención con la que también se coincide.

El logro de la modernización educativa depende substancialmente de una mayor aportación del presupuesto federal para la educación.

Es conveniente que se impulsen enfoques descentralizados que prioricen la atención de problemas y necesidades locales, y hagan eficiente y dinámico al sistema educativo nacional.

Atender con igual interés la urgencia de abatir el rezago educativo en primaria, la educación básica para todos y la demanda en los niveles medió superior y superior.

Resulta plausible el establecimiento de una instancia de concertación de los subsistemas de educación media superior para "la consolidación del nivel y promover la colaboración interinstitucional. “

Es una necesidad insoslayable impulsar el desarrollo científico y tecnológico que responda a las necesidades del desarrollo del país y los retos que representa su inserción en el mercado internacional.

Resulta totalmente adecuado que lo central consista en asegurar cobertura calidad y eficiencia en todos los niveles educativos y que el Programa para la Modernización Educativa represente "la instauración de un espacio para generar reflexión y la acción colectiva en el marco de la ley a fin de asegurar una mejor educación para todos los mexicanos." (ANUIES, 1990: 18-19.)

En cuanto a la estrategia para el mejoramiento y consolidación del sistema nacional de educación superior la ANUIES la estructura atendiendo cinco áreas temáticas: 1. Desarrollo regional de la educación superior. 2. Programas nacionales para el mejoramiento de las funciones de la educación superior. 3. Programas especiales de apoyo al sistema de educación superior, 4. Concertación y vinculación con otros sectores. 5. Financiamiento de la educación superior.

La primera área temática aborda el desarrollo regional a partir de la propia descentralización de la ANUIES, modificando su estructura y normatividad para impulsar y reglamentar el trabajo regional mediante la creación de los Consejos Regionales. Las principales políticas, criterios y mecanismos para el trabajo regional de la ANUIES se concretan en el reconocimiento, por un lado, de la capacidad de autodeterminación de las instituciones y, por otro, 
de la ineludible interdependencia que las complementa y permite su desarrollo. La formación de un auténtico sistema nacional de educación superior parte del reconocimiento de "sistemas" regionales y estatales en los que se reconozcan Y respeten características, intereses y peculiaridades. Debe ser considerado como un "esquema de posibilidades de articulación multidimensional. "En cuanto a los siete programas nacionales para el mejoramiento de las funciones, se proponen, en las áreas prioritarias de formación y actualización del personal académico: mejoramiento del posgrado, la investigación, la educación continua y la administración; difusión de la cultura y extensión de los servicios; y, apoyo al bachillerato. Cinco son los programas especiales que apoya al sistema de educación superior. "Estos programas se caracterizan por implicar acciones de estímulo para el desarrollo del sistema y constituir una palanca que proporcione apoyo a las instituciones, o por explorar alternativas al modelo tradicional de universidad." (ANUIES 1990: 74)

La problemática sobre la que se proponen líneas de acción específicas está referida a: Estímulos y reconocimientos al personal académico; sistema nacional de universidad abierta: sistema nacional de información para la educación superior; red de comunicación y red nacional de bibliotecas.

La ANUIES propone una serie de criterios para concertar acciones con otros agentes responsables de la educación superior, atendiendo a cuatro aspectos fundamentales: la concertación para la coordinación y planeación de la educación superior; la vinculación con la sociedad y con el sector productivo de bienes y servicios; la evaluación de las instituciones y el sistema; la modernización de la normatividad como resultado de la concertación en el sistema, reconociendo los derechos y obligaciones constitucionales de las IES y los gobiernos federal y estatales en esta materia.

El tema del financiamiento de la educación superior la ANUIES lo aborda a través de tres resolutivos generales que tocan puntos de solicitud al gobierno federal y recomendaciones a las IES; de entre ellos sobresalen: Se definan concertadamente entre gobierno federal e IES los "criterios claros y mecanismos eficientes para la asignación de recursos." Presentar en breve plazo (Primer semestre de 1990). Ante los responsables gubernamentales de asignar recursos las propuestas concretas de las IES referidas a suficiencia, oportunidad y equidad en la distribución de los recursos. Solicitar del gobierno federal el incremento, en términos reales, del presupuesto asignado a la educación superior. Que las IES impulsen el desarrollo de acciones para la obtención de ingresos complementarios. Hasta aquí de manera sucinta se han presentado los documentos que se considera que formaron el marco discursivo-propositivo de la modernización de la educación superior; en el 
siguiente apartado se abordará el sentido que desde la perspectiva teórica se puede descubrir en tales propuestas.

\section{Significación y sentido de las propuestas}

Los documentos que bajo ciertas perspectivas son básicos para la modernización de la educación superior presentan una continuidad de contenidos, no sólo por su consecutividad cronológica, sino fundamentalmente como resultado del proceso de modernización adoptado por las IES que antecede a la política del gobierno federal. La modernización de la educación superior como parte de una amplia problemática queda referida en el marco de una doble crisis: la del proyecto universitario en sí mismo y la del proyecto nacional por el desgaste del modelo de desarrollo económico emprendido desde la posguerra. M. Kaplan, al respecto, dice que: "Modernización y universidad son dos términos que han mantenido una prolongada relación en la cual ambos entran bajo el signo de la ambigüedad, en ellas mismas y en sus interrelaciones." (Kaplan, 1989: 197)

¿Cuáles son las ambigüedades más visibles? La universidad, representativa de la educación superior, ha sido visualizada como la «sede del conservadurismo y de la subversión, del tradicionalismo y de la modernización, del intelectual como defensor y legitimador del orden o como crítico predestinado al exilio (interno o externo), de la racionalidad (crítica, ética, práctica).» (Kaplan, 1989: 198)

Los procesos de modernización han sido imposiciones desde el exterior pero también aspiraciones de un futuro deseable. Propuestas desde las élites del poder alejadas de las dinámicas sociales "Han sido prototipos anticipatorios respecto a los supuestos y precondiciones que debería haber tenido, y a los contenidos y resultados que pretendieron tener o prometieron lograr." (Kaplan. 1989: 199)

Jürgen Habermas afirma que el término "moderno" apareció y reapareció en Europa en los períodos en que se formaba “... la conciencia de una nueva época a través de una relación renovada con los antiguos y, además, siempre que la antigüedad se consideraba como un modelo a recuperar a través de alguna clase de imitación," (Habermas, 1988: 20.) pero como él mismo afirma esa atracción que los clásicos del mundo antiguo ejercían sobre "el espíritu de tiempos posteriores" tuvo su primer cambio radical con los ideales de la Ilustración Francesa, en forma particular la idea de ser "moderno", “...dirigiendo la mirada hacia los antiguos, cambió con la creencia, inspirada por la ciencia moderna, en el progreso infinito del conocimiento y el avance in- 
finito hacia la mejoría social y moral Otra forma de conciencia modernista se formó a raíz de ese cambio." (Habermas, 1988)

Así los procesos de modernización están basados en una re-novación de los valores antiguos remotos, o en una in-novación que deshecha los valores antiguos inmediatos, pero siempre como un proceso resultado de una transición de lo antiguo a lo nuevo. La reestructuración mundial del capitalismo iniciada en los últimos años de la década de los setentas colocó a México en los noventas, ante el umbral de "una de esas fronteras siempre fugitivas de la modernidad." Imponer desde arriba y desde afuera, cada vez con mayor autoritarismo, una visión de auge y progreso de los menos cuya generalización abstracta hace aparecer como de la totalidad. Si el gobierno federal anterior asumió como política fundamental una modernización global del país que incluía la modernización de la educación y dentro de ella a la de la educación superior. ¿Cuáles fueron sus características, es decir, su significación y sentido?

La significación y el sentido de las propuestas de modernización de la educación superior salinista se abordarán a partir de áreas conceptuales claves, significantes de las propuestas y orientadores de su sentido, ubicándolas en los ejes de semantización coincidencia-divergencia, presencia- ausencia, manifiesto-oculto. Las áreas conceptuales claves presentes en los documentos básicos y empleadas en este análisis son: Planeación, Evaluación, Funciones sustantivas, Expansión de los servicios, Financiamiento, Normatividad, y Formación de los alumnos.

La Planeación en el PROIDES se planteó como una aspiración a lograrse mediante la mejoría en los procesos, la coordinación de instancias responsables y la cobertura de los ámbitos de actuación. En el Programa de la Modernización Educativa (PME) se proponía fortalecer el sistema de planeación nacional en un marco de concertación en coincidencia con las determinaciones autónomas de las IES, pero haciendo presente de manera encubierta en "los grandes objetivos nacionales en materia de educación superior" el modelo que vincula planeación-financiamiento. En la estrategia para la Consolidación y Desarrollo del Sistema Nacional de la Educación Superior (ECDSNES) se le caracterizó como instancia de concertación en los diferentes niveles, ámbitos e instancias acordadas por la ANUIES para la coordinación con las dependencias federales y estatales.

Rasgo característico en este documento fue la preocupación y puesta en marcha de una organización regional que en el área de la planeación atendería las características, necesidades y perspectivas de desarrollo de las IES con criterios descentralizadores sin perder de vista las líneas generales de las aspiraciones nacionales. El financiamiento de la Educación Superior fue el punto toral de las relaciones Estado: 
Instituciones de Educación Superior. En los documentos de la ANUIES las IES mantuvieron dos demandas permanentes: Incremento de los subsidios en términos reales, criterios y mecanismos eficientes para la asignación de los recursos. El Gobierno Federal en el PME coincidía y aceptaba tales reclamos pero proponía como acciones modernizadoras: "aplicar de manera óptima los recursos disponibles" alentando la opción de búsqueda de ingresos propios. Esta actitud del Estado la hace coincidente con los puntos de vista de Burton R. Clarlk que al hablar de las "universidades efectivas" afirma: "La excelencia competitiva es probablemente, dependiente de la diversificación del financiamiento en las universidades.

Bajo un solo patrón, usualmente el ministerio nacional de educación, es difícil para las universidades distinguirse unas de otras y exhibir iniciativa. Múltiples puentes y diversas líneas de financiamiento dan a las instituciones más libertad para estimular su propia evolución... la mayor garantía de la autonomía universitaria y de su iniciativa es el financiamiento diversificado. Afortunada la universidad que tiene una docena de líneas de financiamiento en lugar de una o dos." (Clark, s/f: 47.) Pero debe quedar claro que no será por la vía de las colegiaturas como se multipliquen realmente las fuentes de financiamiento, aún cuando es indispensable que se analice las disparidades existentes en las colegiaturas de muchas IES. No es aconsejable una homogeneización a nivel nacional pero si modificar las distorsiones actuales, injustificables a todas luces. La evaluación es considerada en los tres documentos como condición indispensable para la planeación; un medio para conocer niveles de rendimiento, productividad eficiencia y calidad del sistema de educación superior en lo general y de cada IES en lo particular.

Existe, en este caso un alto grado de coincidencia en el sentido de la evaluación, sin embargo hay una divergencia por la presencia oculta de significados diferentes. Para el PME debería servir para "depurar y racionalizar sus modalidades, carreras y especialidades" y sus resultados "serán considerados para la determinación de las prioridades de desarrollo de la educación superior", es decir, saber a quien apoyar económicamente y a quien no. Para la ANUIES es el fundamento para la "ratificación o rectificación de políticas, lineamientos, programas y procedimientos" apoyada en "estándares de calidad, rendimiento y efectividad" definidos para el nivel nacional, pero un tanto a la defensiva agrega que su aplicación no debería ser "mecánica directa e indiscriminada a todas las situaciones locales; en cada caso habrá que atender a características, necesidades y grado de desarrollo alcanzado." (ANUIES, 1990: 90) Las funciones sustantivas para una mayor claridad se abordarán específicamente. 
Así las propuestas sobre la docencia se ubican, de manera coincidente en los tres documentos, sobre dos líneas, (formación y actualización de docentes $\mathrm{y}$, actualización de planes de estudio y programas), convergentes en un punto (Mejorar la calidad de los procesos educativos de la educación superior).

Existen, sin embargo, divergencias tanto en el sentido como en la significación en cada documento en lo particular. Tanto en el PROIDES como en el PME la formación y actualización de docentes apunta preferentemente hacia la profesionalización tanto de los catedráticos como de los profesores de carrera. en el PME se introduce un factor que se refuerza en la ECDSNES, el establecimiento de criterios de excelencia en el ejercicio académico.

El sentido oculto de esta propuesta estaría en la transformación de las relaciones de contratación, de gremiales a individuales, y en la valoración de la acción académica promoviendo el "activismo sobre la preparación.”Para el PME la redefinición de los planes de estudio debe tener un sentido esencialmente laboral aún cuando también que evite la excesiva parcelarización de conocimientos y se centren en áreas que tenga posibilidades de desarrollo científico y tecnológico. Para la ANUIES lo prioritario está en la orientación del aprendizaje hacia lo metodológico e instrumental que propicie el desarrollo de aptitudes para el autoaprendizaje y auto información y la preparación para adecuarse a situaciones cambiantes en el conocimiento y en el medio profesional. A la investigación se le da el carácter de "tarea institución permanente" cuyo papel fundamental es "la generación de conocimientos en las ciencias y en las humanidades" cuya modernización "implica un proceso de cambio y transformación de estructuras en el sistema de educación superior."

Las propuestas de los documentos son coincidentes en el sentido y significación de la investigación. Descentralizarla, planearla, vincularla con la docencia y la extensión, difundirla más ampliamente, pero sobre todo apoyarla financieramente mediante la evaluación de sus resultados concretos; son los rasgos significantes manifiestos en las propuestas. Una propuesta relevante del PME y recogida por la ANUIES es la vinculación de los Centros de Investigación con los sectores productivos de bienes y servicios, en ella se puede intuir un sentido si no oculto tampoco manifiesto de ser alternativa de financiamiento.

La extensión de la cultura y los servicios es un área ausente en el PME que reduce sus propuestas a un solo aspecto de esta función: el servicio social; al cual pretende incorporar con el Programa Nacional de Solidaridad. En cambio la ANUIES reconoce que la extensión de la cultura y de los servicios está postergada, con todo y ser cabalmente, "la tarea primordial de la educación superior y el servicio que la sociedad legítimamente espera de ella”. Se le da en las propuestas, un sentido de "función social", resignificándola para 
cada IES al ponerlas en relación directa con las comunidades en donde se asientan, y que conforman su entorno, investigando su historia, divulgando y promoviendo sus productos culturales, emprendiendo servicios de asesoría, gestoría, asistencia y formación.

La expansión de los servicios tiene una presencia manifiesta en los planteamientos de dos de los documentos que conformaron la modernización de la educación superior, existe una clara coincidencia en el sentido asignado a las propuestas en el PROIDES y en el PME: equilibrar, regular y racionalizar el crecimiento son los criterios orientadores significativos. El sentido "modernizador" del crecimiento propuesto por el PME y recogido por la ANUIES estaba en el establecimiento del Sistema Nacional de Universidad Abierta propuesta cuyo significado oculto es el ofrecimiento de un servicio de bajo costo y cuya "clientela" natural sería el rezago o los usuarios de las preparatorias y secundarias abiertas promovidos desde los sexenios anteriores.

El "credencialismo moderno" se concretó en la propuesta de un "sistema de acreditación que reconozca el autodidactismo y los conocimientos adquiridos en la vida productiva." (PEF, 1989: 140.)

La normatividad es una estrategia para la modernización de la educación superior, el sentido con que se propusieron fue divergente; para el PME las iniciativas deberían tender a "lograr una mejor regulación de las actividades de concertación, evaluación, descentralización, fijación de criterios académicos, de financiamiento y de acreditación que el desarrollo de la educación superior está demandando."

(PEF, 1989: 135) La ANUIES consideraba que las normas jurídicas deben ser el resultado de la concertación en el sistema, respetando los derechos y obligaciones constitucionales otorgadas a las IES al reconocerles su autonomía al máximo nivel normativo.

\section{Logros de la modernización de la educación superior}

A partir de las propuestas de modernización de la educación superior descritas y analizadas en los apartados anteriores, analizaremos ahora cuales fueron los logros más significativos y evidentes actualmente y las perspectivas futuras de esos cambios. Se hará desde dos enfoques diversos pero complementarios el primero el análisis de tres "conceptos estelares" en la modernización de la educación superior y en la presentación de dos escenarios uno posible y otro alternativo de la continuidad de esa modernización.

Los conceptos estelares fueron: la competitividad, la privatización y la excelencia. La desintegración del sector paraestatal y la desregulación eco- 
nómica fueron dos manifestaciones claras de la política privatizadora del gobierno salinista y que respondía al desarrollo económico mundial reciente dominado por las corrientes neoliberales. A partir de ella la acción educativa tendió a orientarse hacia el desarrollo del individualismo y la competencia.

La privatización de la educación superior no se dará sólo con el crecimiento de las instituciones atendidas por particulares, sino fundamentalmente en la aceptación acrítica de las sugerencias del sector empresarial para el cambio de planes y programas y la reorientación de la investigación hacia la comercialización de sus productos a través del registro de patentes, (proyectos con esas posibilidades y no otros son los que estuvieron dispuestos a financiar).La productividad como fin educativo conlleva la transmisión de valores como "capacidad" y "desempeño" en el más puro sentido del empresariado hacia sus asalariados.

El individualismo y la competencia son las actitudes que se verían reforzadas en una reforma de la educación superior basada en tales premisas. La productividad como característica de los docentes fue alentada a través de varias acciones específicas, que afectaron directamente a las relaciones laborales y sindicales. Varias universidades empezaron a marginar las acciones gremiales para preferenciar las negociaciones individuales, estableciendo una relación salario-productividad, esto es, a quien más produzca se le ofrece mayores salarios por medio de becas y estímulos económicos

Esto en principio no es malo, lo negativo es el sentido con el que se fue haciendo, por los efectos que ya se están manifestando de manera preocupante. Existen IES donde la preparación y la antigüedad no se consideraron como facto- res de determinación del salario, eran las funciones las que tenían mayor peso. Algunas prestaciones sociales, como el año sabático, se ubicaron dentro de la conceptualización de la productividad. Todo ello encaminado a: Establecer una evaluación permanente del personal académico sin la intervención sindical. Transformar la relación laboral para que la edad y la productividad sustituyan a la preparación, la antigüedad y los contratos colectivos.

Esta transformación de la idea de academia tendió a burocratizar y controlar el trabajo del docente y el investigador y se reflejaron en acciones" compulsivas" por publicar y "ser citado por los cuates" para hacer impactante su productividad. Todo esto afecta la libertad de cátedra y de investigación, que ahora deben ajustarse a las prioridades establecidas y estimuladas por los funcionarios.

La excelencia como tercer concepto estelar en la modernización de la educación superior tiene que ver más con la competitividad que con el logro 
del máximo rigor académico o el dominio efectivo de los contenidos. Ser competitivo en sentido positivo es una actitud que ayuda al ser humano a superar sus propios logros, pero que en el extremo opuesto se vuelve la antítesis de la solidaridad. ¡El enemigo a vencer es el compañero con quien se comparte el cubículo! ¿Cuáles son los escenarios previsibles a partir de tales indicios y de continuar la misma política en actual régimen? en primer lugar, permítase tratar un aspecto relacionado con todo esto. Algunos de los rasgos característicos de la actual revolución industrial-científico-tecnológica son: la ruptura con las viejas disciplinas y el surgimiento de nuevas áreas de conocimiento a las que se les ha llamado" áreas híbridas"; la aparición de nuevos métodos, modelos teóricos y tecnológicos y la reducción del tiempo entre descubrimiento científico y producción industrial. Esta revolución está presente ya en los más diversos campos y sectores de la economía y la sociedad; está transformando las condiciones y posibilidades de la humanidad en los campos social, económico, cultural y político, sobre su poder transformador M. Kaplan afirma: "reclasificadora, concentradora y marginalizante, la mutación en marcha tiende estructuralmente a beneficiar a una minoría de actividades, sectores, grupos, países, regiones, en desmedro de otras y otros que se convierten más o menos paulatinamente en mayorías superfluas o redundanres, en las naciones y en el planeta." (Kaplan, 1989: 214)

Frente a estos retos externos las propuestas de la modernización de la educación superior se ven tímidas y desarticuladas. Ciertamente se plantearon con la intención de acelerar la posibilidad de integrar a México a la Revolución Industrial-Científico- Tecnológica, sin embargo, no se plantearon modos, ni tiempos para lograrlo. Por otro lado, no se cumplieron los "ofrecimientos" de racionalizar la matrícula, reorientar los estudios y siempre hubo una falta de claridad para enfrentar los problemas del financiamiento. Agréguese a lo anterior: el abandono de la visión de largo plazo para ponderar el inmediatismo, la conceptualización basada en la supuesta armonía de los elementos que conforman el sistema y aún la escasa fundamentación para creer en la existencia misma de un sistema nacional de educación superior.

Un escenario alternativo para el desarrollo y modernización de la educación superior estaría dado por al menos tres elementos: la búsqueda de una excelencia académica más que la excelencia competitiva. Su democratización basada en la libre expresión de las ideas y alejada de una hiperpolitización partidista pero sobre todo en el compromiso de formar profesionales polivalentes capaces de adaptarse y responder positivamente a las cambiantes condiciones de la práctica y la generación de nuevos conocimientos. Modernizar y modernizarse con la clara conciencia de vivir un proceso permanente y sistemático y no una moda sexenal u oferta política pasajera. 


\section{Bibliografía}

ANUIES, 1982 Programa integralpara el desarrollo de la Educación Superior, México: ANUIES.

ANUIES.- "Consolidación y desarrollo del sistema nacional de educación superior.", en Revista de la Educación Superior No. 73, ANUIES. México. Enero-Marzo 1990: 18-19.

Burton R., Clark, "El sistema de Educación Superior: Modelos Organizacionales.” en Manuel Barquín Álvarez y Carlos Ornelas. Ornelas (Comps.) Superación Académica y Reforma Universitaria, México: UNAM.

Habermas, Jürgen, 1988: "La modernidad, un proyecto incompleto" en La Posmodernidad, México: Kairos y Colofón S.A.

Kaplan, Marcos. "Modernización y Superación Académica en la Reforma Universitaria.” en Manuel Barquín Alvarez y Carlos Ornelas (Comp.) Superación Académica y Reforma Universitaria, México: UNAM.

PEF Poder Ejecutivo Federal, 1989: Programa de la Modernización Educativa 1989-1994, México: S.P.P. 